\title{
Polarization-dependent quantum beats of four-wave mixing in the Luttinger model for bulk semiconductors
}

\author{
Wenfeng Wang, ${ }^{1,2}$ Klaas Allaart, ${ }^{1, *}$ and Daan Lenstra ${ }^{3}$ \\ ${ }^{1}$ Faculty of Science, VU University Amsterdam, De Boelelaan 1081, 1081HV Amsterdam, The Netherlands \\ ${ }^{2}$ Faculty of Physics and Electronics, Hubei University, Wuhan, China \\ ${ }^{3}$ Faculty of EEMCS, Delft University of Technology, Delft, The Netherlands
}

(Received 22 February 2007; published 17 July 2007)

\begin{abstract}
We examine the description of quantum beats in four-wave mixing with bulk semiconductors within the framework of the Luttinger-Kohn model. An analytic expression for their dependence on the relative linear polarization of pump and probe is derived, taking only the band structure and coherent interaction of the light waves with the semiconductor medium into account. Herewith all features seen in experiments are very well reproduced, e.g., the vanishing of the beats for an angle $\theta_{0} \approx 76^{\circ}$ between the polarizations of pump and probe. Therefore, as opposed to general belief based on earlier theoretical work, no ad hoc exciton-exciton Coulomb interaction has to be invoked to describe the observed phase and magnitude of the quantum beats for copolarized or for cross-polarized test and pump pulses.
\end{abstract}

DOI: 10.1103/PhysRevB.76.035206

\section{INTRODUCTION}

Polarization-dependent four-wave mixing (FWM) quantum beats ${ }^{1-11}$ have been observed after simultaneous excitations of two optical transitions, associated with heavy hole and light hole. The signal magnitude and its beat phase were found to depend on the relative linear polarization of the pump and test pulses. This phenomenon has been analyzed by applying semiconductor Bloch equations (SBE) for excitations in a six-band model with broad spectrum pump-probe pulses. ${ }^{1,2}$ These theoretical studies did not reproduce essential details of the observations. For instance, they predicted identical FWM intensities for the two cases: pump and probe having either parallel or perpendicular linear polarizations. Subsequently, a successful explanation was claimed to be given by the biexciton theory, ${ }^{3,5-11}$ invoking a phenomenological exciton-exciton coupling attributed to Coulombic interactions. However, it has remained obscure why the SBE would fail for the phenomenon in a semiconductor.

In the present work, we shall completely neglect the Coulomb interaction between the charge carriers, which means that no exciton-exciton Coulomb interaction is introduced. We just solve the Heisenberg equations of motion for the dipole to third order in the light-matter interaction only. This is done within the framework of the Luttinger model in order to correctly account for the structure of the band wave functions. An analytic expression for the polarization dependence of the quantum beats is then derived and shown to represent surprisingly well all features seen in experiments. We shall therefore conclude that FWM quantum beats can be explained as a purely coherent light-matter interaction effect, without invoking a phenomenological exciton-exciton interaction.

In the Luttinger model for III-V semiconductors, one has, ignoring split-off bands, for the lattice periodic functions of the valence bands the degenerate heavy-hole states $|h 1 \vec{k}\rangle,|h 2 \vec{k}\rangle$ with energy $E_{h, k}$ and the light-hole states $|l 1 \vec{k}\rangle,|l 2 \vec{k}\rangle$ with energy $E_{l, k}$. These are superpositions, depending on the Bloch vector $\vec{k}$, of the $p$-like functions ${ }^{12}$
PACS number(s): 78.66.Fd, 42.50.Md, 42.65.- $\mathrm{k}$

$$
\begin{gathered}
|3 / 2,3 / 2\rangle=-\sqrt{1 / 2}(|X \uparrow\rangle+i|Y \uparrow\rangle), \\
|3 / 2,1 / 2\rangle=-\sqrt{1 / 6}(|X \downarrow\rangle+i|Y \downarrow\rangle)+\sqrt{2 / 3}|Z \uparrow\rangle, \\
|3 / 2,-1 / 2\rangle=\sqrt{1 / 6}(|X \uparrow\rangle-i|Y \uparrow\rangle)+\sqrt{2 / 3}|Z \downarrow\rangle, \\
|3 / 2,-3 / 2\rangle=\sqrt{1 / 2}(|X \downarrow\rangle-i|Y \downarrow\rangle),
\end{gathered}
$$

and can be written in a compact matrix multiplication form as $^{13}$

$$
\left(\begin{array}{c}
|h 1 \vec{k}\rangle \\
|h 2 \vec{k}\rangle \\
|l 1 \vec{k}\rangle \\
|l 2 \vec{k}\rangle
\end{array}\right)=\frac{1}{\sqrt{N}}\left(\begin{array}{cccc}
-b & R & 0 & -c^{*} \\
-c & 0 & R & b^{*} \\
R & b^{*} & c^{*} & 0 \\
0 & c & -b & R
\end{array}\right)\left(\begin{array}{c}
|3 / 2,3 / 2\rangle \\
|3 / 2,1 / 2\rangle \\
|3 / 2,-1 / 2\rangle \\
|3 / 2,-3 / 2\rangle
\end{array}\right),
$$

with eigenenergies $E_{i}, i=h, l, R=H_{h}-E_{h}=E_{l}-H_{l}$, and $N$ $=R^{2}+|c|^{2}+|b|^{2}$. Here, we use the conventional notations ${ }^{12}$

$$
\begin{gathered}
b=\sqrt{3} \bar{\gamma} \hbar^{2}\left(m_{0}\right)^{-1}\left(k_{x}-i k_{y}\right) k_{z}, \\
c=\sqrt{3} \bar{\gamma} \hbar^{2}\left(2 m_{0}\right)^{-1}\left[\left(k_{x}^{2}-k_{y}^{2}\right)-2 i k_{x} k_{y}\right], \\
H_{h}=-\hbar^{2}\left(2 m_{0}\right)^{-1}\left[\gamma_{1} k^{2}-\gamma_{2}\left(2 k_{z}^{2}-k_{\perp}^{2}\right)\right], \\
H_{l}=-\hbar^{2}\left(2 m_{0}\right)^{-1}\left[\gamma_{1} k^{2}+\gamma_{2}\left(2 k_{z}^{2}-k_{\perp}^{2}\right),\right],
\end{gathered}
$$

with $\gamma_{1}, \gamma_{2}$, and $\bar{\gamma}$ the empirical parameters of the Luttinger model. The dependence of these wave functions (2) on the Bloch vector $\vec{k}$ makes the situation in a semiconductor essentially different from that in atomic systems. The states of the conduction band $|c 1 \vec{k}\rangle,|c 2 \vec{k}\rangle$ are, for small momentum $\vec{k}$, approximated by the two spin-degenerate $s$-wave-like functions

$$
|c 1 \vec{k}\rangle=|S \uparrow\rangle, \quad|c 2 \vec{k}\rangle=|S \downarrow\rangle .
$$




\section{MODEL}

The light-matter interaction is described in dipole approximation as $-e \overrightarrow{\mathcal{E}}(t) \cdot \vec{r}$. We consider linearly polarized laser light in the $x-y$ plane. The $x$ component of the dipole operator that couples to $x$-polarized light is expressed as a superposition of particle-hole operators for the band states with $\vec{k}$ dependent coefficients. Explicitly, omitting for a moment the label $\vec{k}$ on the operators (also, the coefficients are $\vec{k}$ dependent) and in units of $M=\langle S|x| X\rangle$,

$$
\begin{aligned}
x= & \sum_{\vec{k}}\left[a_{c 1}^{\dagger} a_{h 1}(-b u)+a_{c 1}^{\dagger} a_{h 2}(w R-c u)+a_{c 1}^{\dagger} a_{l 1}\left(u R+w c^{*}\right)\right. \\
& +a_{c 1}^{\dagger} a_{l 2}(-w b)+a_{c 2}^{\dagger} a_{h 1}\left(u c^{*}-w R\right)+a_{c 2}^{\dagger} a_{h 2}\left(-u b^{*}\right) \\
& \left.+a_{c 2}^{\dagger} a_{l 1}\left(-w b^{*}\right)-a_{c 2}^{\dagger} a_{l 2}(w c+u R)\right]+ \text { H.c., } \\
y= & \sum_{\vec{k}} i\left[a_{c 1}^{\dagger} a_{h 1}(-b u)-a_{c 1}^{\dagger} a_{h 2}\left(w R_{h}+c u\right)+a_{c 1}^{\dagger} a_{l 1}\left(u R_{l}-w c^{*}\right)\right. \\
& +a_{c 1}^{\dagger} a_{l 2}(w b)-a_{c 2}^{\dagger} a_{h 1}\left(w R_{h}+u c^{*}\right)+a_{c 2}^{\dagger} a_{h 2}\left(u b^{*}\right) \\
& \left.+a_{c 2}^{\dagger} a_{l 1}\left(-w b^{*}\right)+a_{c 2}^{\dagger} a_{l 2}\left(-w c+u R_{l}\right)\right]+ \text { H.c., }
\end{aligned}
$$

with $u=-\sqrt{1 / 2}$ and $w=\sqrt{1 / 6}$.

In four-wave mixing experiments, the incident light fields are a pump field $\overrightarrow{\mathcal{E}}_{p}$ and a much weaker probe (testing) field $\overrightarrow{\mathcal{E}}_{t}$. We assume that the electric field strength is composed of a (strong) pulse $\overrightarrow{\mathcal{E}}_{p}(\vec{r}, t)$, during a short time $\delta$ around $t=0$, and a test pulse $\overrightarrow{\mathcal{E}}_{t}(\vec{r}, t)$, preceding it, during a short time span $\delta$ around $t=-\tau$.

$$
\begin{aligned}
\overrightarrow{\mathcal{E}}(\vec{r}, t)= & \hat{\mathcal{E}}_{p}\left[\widetilde{\mathcal{E}}_{p}(t) \exp \left(i \vec{q}_{p} \cdot \vec{r}-\omega t\right)+\text { c.c. }\right] \\
& +\hat{\mathcal{E}}_{t}\left[\widetilde{\mathcal{E}}_{t}(t) \exp \left(i \vec{q}_{t} \cdot \vec{r}-\omega t\right)+\text { c.c. }\right]
\end{aligned}
$$

The envelop functions $\widetilde{\mathcal{E}}_{p}$ and $\widetilde{\mathcal{E}}_{t}$ are smoothly and slowly varying in time and satisfy

$$
\left|\widetilde{\mathcal{E}}_{p}(t)\right| \approx 0 \quad \text { for }|t|>\delta, \quad\left|\widetilde{\mathcal{E}}_{t}(t)\right| \approx 0 \text { for }|t+\tau|>\delta .
$$

The pulse lengths must be long enough to justify the distinction between resonant and nonresonant terms, therefore, several times the oscillation period of the fields:

$$
2 \delta \gg \frac{2 \pi}{\omega} \equiv T_{F} .
$$

On the other hand, they should be short enough for the delay time between the two pulses to be well defined on the time scale of the beat time:

$$
2 \delta \ll \frac{2 \pi}{\omega_{h}-\omega_{l}} \equiv T_{B} .
$$

Otherwise, the whole beat phenomenon will be washed out by the average over a too broad range of delay times. In the experiments, ${ }^{2}$ one observed $T_{B} \approx 1 \mathrm{ps}$, while $T_{F}$ is a few femtoseconds. So, pulses of roughly $100 \mathrm{fs}$ are quite suitable.

We consider the case that the pump pulse $\overrightarrow{\mathcal{E}}_{p}$ is linearly polarized in $x$ direction, while the linear polarization of the test pulse makes an angle $\theta_{0}$ with that of the pump pulse: $\hat{\mathcal{E}}_{t}=\hat{x} \cos \theta_{0}+\hat{y} \sin \theta_{0}$.

The detected intensity of the FWM signal is ${ }^{2}$

$$
I_{\mathrm{FWM}} \propto \int d t\left|\overrightarrow{\mathcal{P}}_{F W M}(t)\right|^{2} .
$$

Here, $\overrightarrow{\mathcal{P}}_{F W M}$ is the component of the third order polarization of second order in $\overrightarrow{\mathcal{E}}_{p}$ and first order in $\overrightarrow{\mathcal{E}}_{t}$ :

$$
\overrightarrow{\mathcal{P}}_{F W M} \propto \exp \left[i\left(2 \vec{q}_{p}-\vec{q}_{t}\right) \cdot \vec{r}\right] .
$$

The Hamiltonian is the independent particle part,

$$
H_{0}=\sum_{i, \vec{k}}\left(E_{c i \vec{k}} a_{c i \vec{k}}^{\dagger} a_{c i \vec{k}}+E_{h i \vec{k}} a_{h i \vec{k}}^{\dagger} a_{h i \vec{k}}+E_{l i \vec{k}} a_{l i \vec{k}}^{\dagger} a_{l i \vec{k}}\right),
$$

plus the interaction of the carriers with the light field,

$$
H_{I}=-e\left[\mathcal{E}_{x}(\vec{r}, t) \cdot x+\mathcal{E}_{y}(\vec{r}, t) \cdot y\right],
$$

with $x$ and $y$ the operators of Eqs. (5) and (6). The polarization is obtained by solving the Heisenberg equations of motion,

$$
\frac{d}{d t} \overrightarrow{\mathcal{P}}=\frac{1}{i \hbar}\left[\overrightarrow{\mathcal{P}}, H_{0}+H_{I}\right]_{-},
$$

to third order in the light-matter interaction. For convenience of the notation, we introduce

$$
\omega_{h k}=\left(E_{c i \vec{k}}-E_{h i \vec{k}}\right) / \hbar \quad \text { and } \omega_{l k}=\left(E_{c i \vec{k}}-E_{l i \vec{k}}\right) / \hbar,
$$

which are independent of the band indices $i$ due to the twofold degeneracy of the bands and independent of the direction of $\vec{k}$ due to the assumed intrinsic isotropy of the bulk semiconductor.

\section{ANALYSIS}

To illustrate some key features of the calculation, we first consider the solution of a particle-hole operator at a time $t^{\prime \prime}$, after the passage of the first (test) pulse $\overrightarrow{\mathcal{E}}_{t}$ only. The integrated equation of motion gives, for band indices $r=1,2, q$ $=1,2$,

$$
\begin{aligned}
a_{c r \vec{k}}^{\dagger}\left(t^{\prime \prime}\right) a_{h q \vec{k}}\left(t^{\prime \prime}\right)= & \frac{e}{i \hbar} \int_{-\infty}^{t^{\prime \prime}} d t^{\prime}\left[\widetilde{\mathcal{E}}_{t}\left(t^{\prime}\right) e^{i\left(\vec{q}_{t} \vec{r}-\omega t^{\prime}\right)}+\widetilde{\mathcal{E}}_{t}^{*}\left(t^{\prime}\right) e^{-i\left(\vec{q}_{t} \cdot \vec{r}-\omega t^{\prime}\right)}\right] e^{i \omega_{h k}\left(t^{\prime \prime}-t^{\prime}\right)} \sum_{s=1}^{2}\left[\left\langle h s \vec{k}\left|\hat{\mathcal{E}}_{t} \cdot \vec{r}\right| c r \vec{k}\right) a_{h s \vec{k}}^{\dagger}(-\infty) a_{h q \vec{k}}(-\infty)\right. \\
& \left.-\left\langle h q \vec{k}\left|\hat{\mathcal{E}}_{t} \cdot \vec{r}\right| c s \vec{k}\right\rangle a_{c r \vec{k}}^{\dagger}(-\infty) a_{c s \vec{k}}(-\infty)\right] .
\end{aligned}
$$


As the pulse is supposed to be sufficiently long, Eq. (9), the nonresonant term with rapidly oscillating integrand factor $\exp \left\{i\left(\omega_{h k}+\omega\right) t^{\prime}\right\}$ is discarded. Without initial correlations, the statistical expectation values of the operators are

$$
\begin{aligned}
& \left\langle a_{h s \vec{k}}^{\dagger}(-\infty) a_{h q \vec{k}}(-\infty)\right\rangle=\delta_{s q} n_{h \vec{k}} \approx 1, \\
& \left\langle a_{c r \vec{k}}^{\dagger}(-\infty) a_{c s \vec{k}}(-\infty)\right\rangle=\delta_{r s} n_{c \vec{k}} \approx 0 .
\end{aligned}
$$

We therefore obtain

$$
\begin{aligned}
a_{c r k}^{\dagger}\left(t^{\prime \prime}\right) a_{h q \vec{k}}\left(t^{\prime \prime}\right) \approx & \frac{e}{i \hbar} e^{-i \vec{q}_{t} \cdot \vec{r}}\left\langle h q \vec{k}\left|\hat{\mathcal{E}}_{t} \cdot \vec{r}\right| c r \vec{k}\right\rangle e^{i \omega_{h k^{\prime \prime}}} \\
& \times \int_{-\infty}^{t^{\prime \prime}} d t^{\prime} \widetilde{\mathcal{E}}_{t}^{\star}\left(t^{\prime}\right) e^{i\left(\omega-\omega_{h k}\right) t^{\prime}} .
\end{aligned}
$$

Because of conditions (8) and (10), we may also apply the approximation

$$
\int d t^{\prime} \widetilde{\mathcal{E}}_{t}^{\star}\left(t^{\prime}\right) e^{i\left(\omega-\omega_{h k}\right) t^{\prime}} \approx e^{-i\left(\omega_{l k}-\omega_{h k}\right) \tau} \int d t^{\prime} \widetilde{\mathcal{E}}_{t}^{\star}\left(t^{\prime}\right) e^{i\left(\omega-\omega_{l k}\right) t^{\prime}} .
$$

In the same fashion, the action of the pump pulse to second order in $\overrightarrow{\mathcal{E}}_{p}$ is treated as a twofold integral over times $t^{\prime \prime}$ and $t^{\prime \prime \prime}$ centered around $t=0$. We further introduce a relaxation or dephasing constant $\lambda$ for the polarization, which simulates the effect of Coulomb collisions. Treating all particle-hole operators in Eqs. (5) and (6) in this way, we obtain, for the FWM component of the polarization $\overrightarrow{\mathcal{P}}=\hat{x} x+\hat{y} y$,

$$
\begin{aligned}
\vec{P}_{F W M}(t) \approx & \frac{e^{3}}{i \hbar^{3}} e^{i\left(2 \vec{q}_{p}-\vec{q}_{t}\right) \cdot r} \sum_{|k|} e^{i\left(\omega_{h k^{-\omega)}}\right)} \int_{t_{0}}^{t} d t^{\prime \prime \prime} \widetilde{\mathcal{E}}_{p}\left(t^{\prime \prime \prime}\right) \\
& \times \int_{t_{0}}^{t^{\prime \prime \prime}} d t^{\prime \prime} \widetilde{\mathcal{E}}_{p}\left(t^{\prime \prime}\right) \int_{-\infty}^{t^{\prime \prime}} d t^{\prime} \widetilde{\mathcal{E}}_{t}^{\star}\left(t^{\prime}\right) e^{-\lambda(t+\tau)} \\
& \times\left\{e^{-i \omega_{h k^{t}}[}\left[\vec{A}+\vec{B} e^{i\left(\omega_{h k^{-}} \omega_{l k}\right) \tau}\right]\right. \\
& \left.+e^{-i \omega_{l k} t}\left[\vec{B}+\vec{C} e^{i\left(\omega_{h k^{-}}-\omega_{l k}\right) \tau}\right]\right\},
\end{aligned}
$$

with

$$
\begin{aligned}
\vec{A}= & 2 \int d \Omega_{\vec{k}} \sum_{i j r q}\langle h i, \vec{k}|\vec{r}| c j, \vec{k}\rangle\langle c j, \vec{k}|x| h r, \vec{k}\rangle\left\langle h r, \vec{k}\left|\hat{\mathcal{E}}_{t} \cdot \vec{r}\right| c q, \vec{k}\right\rangle \\
& \times\langle c q, \vec{k}|x| h i, \vec{k}\rangle, \\
\vec{B}= & 2 \int d \Omega_{\vec{k}} \sum_{i j r q}\langle h i, \vec{k}|\vec{r}| c j, \vec{k}\rangle\langle c j, \vec{k}|x| l r, \vec{k}\rangle\left\langle l r, \vec{k}\left|\hat{\mathcal{E}}_{t} \cdot \vec{r}\right| c q, \vec{k}\right\rangle \\
& \times\langle c q, \vec{k}|x| h i, \vec{k}\rangle, \\
\vec{C}= & 2 \int d \Omega_{\vec{k}} \sum_{i j r q}\langle l i, \vec{k}|\vec{r}| c j, \vec{k}\rangle\langle c j, \vec{k}|x| l r, \vec{k}\rangle\left\langle l r, \vec{k}\left|\overrightarrow{\mathcal{E}}_{t} \cdot \vec{r}\right| c q, \vec{k}\right\rangle \\
& \times\langle c q, \vec{k}|x| l i, \vec{k}\rangle .
\end{aligned}
$$

Here, we split the summation over $\vec{k}$ into a summation over the modulus $|k|$ and integration over its angles. The latter determines the dependence of the FWM intensity on the angle between the polarizations of the pulses represented by the factor $\hat{\mathcal{E}}_{t} \cdot \vec{r}$. The matrix elements of the dipole components are the coefficients in Eqs. (5) and (6) and the angle integration can easily be done analytically. The detected intensities of the FWM signal then become

$$
\begin{aligned}
I_{\|}= & F e^{-2 \lambda \tau} \frac{1}{2 \lambda} \sum_{|k|}\left\{208+192 \cos \left(\omega_{h k}-\omega_{l k}\right) \tau\right. \\
& +\frac{4 \lambda^{2}}{4 \lambda^{2}+\left(\omega_{h k}-\omega_{l k}\right)^{2}}\left[192+208 \cos \left(\omega_{h k}-\omega_{l k}\right) \tau\right] \\
& \left.+\frac{2 \lambda\left(\omega_{h k}-\omega_{l k}\right)}{4 \lambda^{2}+\left(\omega_{h k}-\omega_{l k}\right)^{2}} 40 \sin \left(\omega_{h k}-\omega_{l k}\right) \tau\right\}, \\
I_{\perp}= & F e^{-2 \lambda \tau} \frac{1}{2 \lambda} \sum_{|k|}\left\{37-12 \cos \left(\omega_{h k}-\omega_{l k}\right) \tau\right. \\
& +\frac{4 \lambda^{2}}{4 \lambda^{2}+\left(\omega_{h k}-\omega_{l k}\right)^{2}}\left[-12+37 \cos \left(\omega_{h k}-\omega_{l k}\right) \tau\right] \\
& \left.-\frac{2 \lambda\left(\omega_{h k}-\omega_{l k}\right)}{4 \lambda^{2}+\left(\omega_{h k}-\omega_{l k}\right)^{2}} 17.5 \sin \left(\omega_{h k}-\omega_{l k}\right) \tau\right\},
\end{aligned}
$$

in which the factor $F$ contains the modulus squared of the prefactor in Eq. (20) with integrals over the envelop functions of the pulses. The summation (integration) extends over the range of $|k|$ values involved in the excitations that are in experiments identified as heavy-hole and light-hole excitons. As we do not describe excitons and their widths explicitly, we adopt a summation over beat frequencies $\omega_{h k}-\omega_{l k}$ in a small range of $|k|$ values. The value of the relaxation parameter $\lambda$ can also be clearly read off from the experimental decay of the FWM signal as a function of the delay time $\tau$. With this, one verifies that the first two terms in expressions (21) and (22) dominate. For an angle $\theta_{0}$ between both polarizations, we therefore find the approximation

$$
\begin{gathered}
I\left(\theta_{0}\right) \propto e^{-2 \lambda \tau} \sum_{|k|}\left\{\left[208+192 \cos \left(\omega_{h k}-\omega_{l k}\right) \tau\right] \cos ^{2} \theta_{0}\right. \\
\left.+\left[37-12 \cos \left(\omega_{h k}-\omega_{l k}\right) \tau\right] \sin ^{2} \theta_{0}\right\} .
\end{gathered}
$$

This is our main result. Due to the summation of $|k|$ in a small region, the beat frequency will not be completely sharp, leading to some smoothing as a function of the delay time $\tau$, resulting in Fig. 1. The figure and Eq. (23) show three features that are also observed in experiment. ${ }^{2}$ Firstly, the FWM signal is stronger in the case of parallel polarization than in case of cross polarization by roughly a factor of 5 , in good agreement with the experiment of Bennhardt et al. ${ }^{2}$ and in contrast to earlier theoretical expressions, ${ }^{1}$ which predicted equal strength in both cases. Secondly, we do indeed find a beat behavior as a function of the delay time which has a maximum at zero delay time for the case of parallel polarization and an opposite oscillating behavior for orthogonal polarization. Thirdly, we find that the beats are more pronounced for parallel polarization than for orthogonal polarization. The beats vanish for $\tan ^{2}\left(\theta_{0}\right)=16$, that is, for $\theta_{0}$ $\approx 76^{\circ}$. In view of the various approximations made, this is in remarkable agreement with the observations in Ref. 2 All 


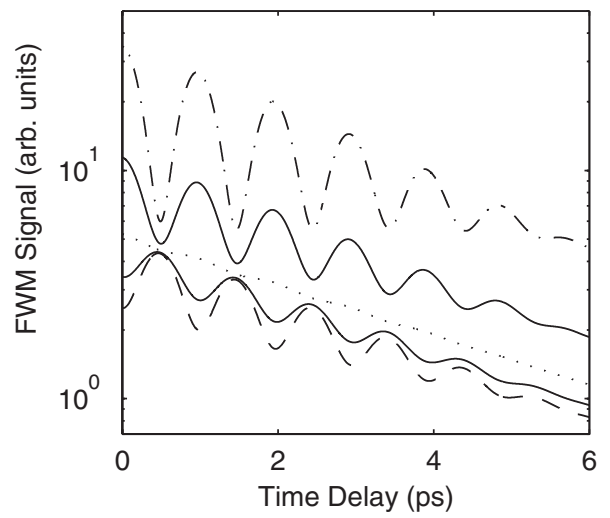

FIG. 1. Dependence of quantum beats in four wave-mixing (FWM) on the relative (linear) polarization angle $\theta_{0}$ between pump and probe pulses, within the Luttinger-Kohn model. Plotted is the integrated FWM signal as a function of delay time $\tau$ between probe and pump, assuming a beat period of 1 ps. The (upper) dash-dotted line for is for parallel polarization, $\theta_{0}=0$, and the (lowest) dashed line is for orthogonal polarization, $\theta_{0}=\pi / 2$. The beats vanish for $\theta_{0}=76^{\circ}$ (dotted line); the full lines are for intermediate angles $0.32 \pi$ (upper) and $0.45 \pi$ (lower).

these features seen in experiments are reflected in Eq. (23) without invoking other mechanisms than just the coupling of the carrier (polarization) dynamics with the light fields. This is in contrast to, for instance, Ref. 2, where the data were interpreted by introducing a coupling parameter ascribed to disorder.
One may remark that the analytic derivation given here involves the Luttinger-Kohn model wave functions (2) for isotropic semiconductor, whereas experiments were done with quantum well material. In the latter, the wave functions may be modified by strain ${ }^{13}$ and by a confining potential. The similarity between our analytic result [Eq. (23)] and the experimental findings indicates, however, that the essentials of the $\vec{k} \cdot \vec{p}$ Luttinger-Kohn model structure of heavy-hole and light-hole wave functions still play an important role in quantum wells with a thickness of a few tens of nanometers.

\section{CONCLUSION}

We have examined the description of quantum beats in four-wave mixing (FWM) with bulk semiconductors within the framework of the Luttinger-Kohn model. An analytic expression for their dependence on the relative linear polarization of pump and probe is derived, taking only the band structure and coherent interaction of the light waves with the semiconductor medium into account. Herewith, all features seen in experiments are very well reproduced. Therefore, as opposed to general belief based on earlier theoretical work, no ad hoc exciton-exciton Coulomb interaction has to be invoked to describe the observed phase and magnitude of the FWM quantum beats.

\section{ACKNOWLEDGMENT}

The authors acknowledge financial support by the Freeband Communication Impulse of the technology programme of the Netherlands' Ministry of Economic Affairs. *allaart@ nat.vu.nl

${ }^{1}$ S. Schmitt-Rink, D. Bennhardt, V. Heuckeroth, P. Thomas, P. Haring, G. Maidorn, H. Bakker, K. Leo, D. S. Kim, J. Shah, and K. Kohler, Phys. Rev. B 46, 10460 (1992).

${ }^{2}$ D. Bennhardt, P. Thomas, R. Eccleston, E. J. Mayer, and J. Kuhl, Phys. Rev. B 47, 13485 (1993).

${ }^{3}$ E. J. Mayer, G. O. Smith, V. Heuckeroth, J. Kuhl, K. Bott, A. Schulze, T. Meier, D. Bennhardt, S. W. Koch, P. Thomas, R. Hey, and K. Ploog, Phys. Rev. B 50, 14730 (1994).

${ }^{4}$ T. Aoki, G. Mohs, T. Ogasawara, R. Shimano, M. KuwataGonokami, and A. Yamaguchi, Opt. Express 1, 364 (1997).

${ }^{5}$ T. Aoki, G. Mohs, M. Kuwata-Gonokami, and A. A. Yamaguchi, Phys. Rev. Lett. 82, 3108 (1999).

${ }^{6}$ D. J. Lovering, R. T. Phillips, G. J. Denton, and G. W. Smith, Phys. Rev. Lett. 68, 1880 (1992).
${ }^{7}$ H. P. Wagner, A. Schatz, W. Langbein, J. M. Hvam, and A. L. Smirl, Phys. Rev. B 60, 4454 (1999).

${ }^{8}$ J. Ishi, H. Kunugita, K. Ema, T. Ban, and T. Kondo, Phys. Rev. B 63, 073303 (2001).

${ }^{9}$ K. Bott, O. Heller, D. Bennhardt, S. T. Cundiff, P. Thomas, E. J. Mayer, G. O. Smith, R. Eccleston, J. Kuhl, and K. Ploog, Phys. Rev. B 48, 17418 (1993).

${ }^{10}$ T. Saiki, M. Kuwata-Gonokami, T. Matsusue, and H. Sakaki, Phys. Rev. B 49, 7817 (1994).

${ }^{11}$ T. Saiki, M. K.-G. Ohkawa, and T. Mitsuyu, Solid State Commun. 95, 679 (1995).

${ }^{12}$ W. W. Chow, S. W. Koch, and M. Sargent III, SemiconductorLaser Physics (Springer, Berlin, 1994).

${ }^{13}$ W. Wang, K. Allaart, and D. Lenstra, Phys. Rev. B 74, 073201 (2006). 